\title{
CG6015 controls spermatogonia transit-amplifying divisions by epidermal growth factor receptor signaling in Drosophila testes
}

\author{
Jun Yu $\mathbb{D}^{1}$, Qianwen Zheng' ${ }^{2}$, Zhiran $\mathrm{Li}^{1}$, Yunhao $\mathrm{Wu}^{1}$, Yangbo $\mathrm{Fu}^{1}$, Xiaolong $\mathrm{Wu}^{1}$, Dengfeng Lin', Cong Shen ${ }^{3}$, \\ Bo Zheng (i) $^{3}$ and Fei Sun (1) ${ }^{1}$
}

\begin{abstract}
Spermatogonia transit-amplifying (TA) divisions are crucial for the differentiation of germline stem cell daughters. However, the underlying mechanism is largely unknown. In the present study, we demonstrated that CG6015 was essential for spermatogonia TA-divisions and elongated spermatozoon development in Drosophila melanogaster. Spermatogonia deficient in CG6015 inhibited germline differentiation leading to the accumulation of undifferentiated cell populations. Transcriptome profiling using RNA sequencing indicated that CG6015 was involved in spermatogenesis, spermatid differentiation, and metabolic processes. Gene Set Enrichment Analysis (GSEA) revealed the relationship between CG6015 and the epidermal growth factor receptor (EGFR) signaling pathway. Unexpectedly, we discovered that phosphorylated extracellular regulated kinase (dpERK) signals were activated in germline stem cell (GSC)-like cells after reduction of CG6015 in spermatogonia. Moreover, Downstream of raf1 (Dsor1), a key downstream target of EGFR, mimicked the phenotype of CG6015, and germline dpERK signals were activated in spermatogonia of Dsor1 RNAi testes. Together, these findings revealed a potential regulatory mechanism of CG6015 via EGFR signaling during spermatogonia TA-divisions in Drosophila testes.
\end{abstract}

\section{Introduction}

The balance between proliferation and differentiation is crucial for germline homeostasis and is tightly controlled by the stem cell niche and spermatogonia transitamplifying (TA) divisions ${ }^{1}$. In Drosophila melanogaster testes, the germline differentiation program must coordinate with germline stem cells (GSCs) self-renewal, and meiosis must take place at the appropriate time during spermatogonia TA-divisions ${ }^{2}$. However, the mechanisms

Correspondence: Jun Yu (yujun9117@126.com) or

Bo Zheng (mansnoopy@163.com) or Fei Sun (sunfei@ntu.edu.cn)

${ }^{1}$ Institute of Reproductive Medicine, School of Medicine, Nantong University,

Nantong, China

${ }^{2}$ Department of Gynecology, the Affiliated Hospital of Jiangsu University,

Jiangsu University, Zhenjiang, China

Full list of author information is available at the end of the article

These authors contributed equally: Jun Yu, Qianwen Zheng, Zhiran Li,

Yunhao Wu

Edited by LM Martins regulating the homeostasis between proliferation and differentiation during spermatogonia TA-divisions are not fully understood.

Regulation of germ cell differentiation has been implicated to involve a number of classic signaling pathways. For instance, germ cell differentiation is repressed by Bone Morphogenetic Protein (BMP) signaling. Decapentaplegic (Dpp) and Glass Bottom Boat (Gbb), which are secreted by somatic hub cells and cyst cells, activate BMP signaling in GSCs and inhibit bam expression to prevent GSC differentiation ${ }^{3,4}$. The Bam protein is critical for the switch from proliferation to meiotic differentiation $^{5}$. Importantly, an aberrant reduction in the Bam level arrests the differentiation pathway and generates extra GSC-like cells ${ }^{6}$.

The Epidermal Growth Factor Receptor (EGFR) signaling pathway is involved in cancer, proliferation, and cell fate determination ${ }^{1,7,8}$. Interestingly, aberrant EGFR

\section{(c) The Author(s) 2021}

(c) (i) Open Access This article is licensed under a Creative Commons Attribution 4.0 International License, which permits use, sharing, adaptation, distribution and reproduction cc) in any medium or format, as long as you give appropriate credit to the original author(s) and the source, provide a link to the Creative Commons license, and indicate if changes were made. The images or other third party material in this article are included in the article's Creative Commons license, unless indicated otherwise in a credit line to the material. If material is not included in the article's Creative Commons license and your intended use is not permitted by statutory regulation or exceeds the permitted use, you will need to obtain permission directly from the copyright holder. To view a copy of this license, visit http://creativecommons.org/licenses/by/4.0/. 
signaling results in abnormal organ formation and tumorigenesis ${ }^{9,10}$. EGFR signaling is also essential for the self-renewal of follicle stem cells (FSCs) through the LKB1-AMPK pathway, while Notch signaling promotes differentiation of the prefollicle cells in the Drosophila ovary $^{11,12}$. Groucho could be phosphorylated by EGFR signaling and inhibits Notch to trigger FSCs differentiation $^{13}$. Meanwhile, extracellular regulated kinase (ERK) regulates high mitochondrial membrane potential, and the phosphorylated form of ERK (dpERK) is increased in drp1 mutant posterior follicle cells in the Drosophila ovary $^{14}$. Moreover, multiple studies have demonstrated that EGFR in somatic cells is critically important for the balance of self-renewal and differentiation in Drosophila testes $^{1,15,16}$. Germline specific ligand-EGF catalyzes the activation of the EGFR signaling pathway by somatic receptors, leading to the aggregation of adapter protein like Grb2 and the guanine exchange factor Sos. Membrane associated Ras protein is triggered by Sos, and thereby activates the phosphorylation of MAPK cascades, which are consists of three kinases including Raf, MEK (Downstream of raf1, Dsor1) and ERK (rolled, rl). Ras phosphorylates MEK, and then ERK is dually phosphorylated by MEK. dpERK in turn phosphorylates and modulates a wide range of substrates both in cytoplasm and nucleus of cells ${ }^{17-19}$. Somatic EGFR plays key roles in the enclosure of GSCs and triggers the early steps of germline differentiation in Drosophila testes ${ }^{20}$. Downregulation of EGFR in cyst cells affects synchronized spermatogonia TA-divisions and the localization of Armadillo on the cyst membrane, while somatic EGFR activation is not required to induce Bam expression ${ }^{21}$.

In the present study, we investigated the transition from proliferation to differentiation during spermatogonia TAdivisions in Drosophila testes. Previously, a large-scale RNA interference (RNAi) screen identified a series of genes required for GSC maintenance ${ }^{22}$, among which a novel GSC regulator, CG6015, which might assist in the mRNA splicing process. The results of the present study show that CG6015 is a key module for spermatogonia TA-divisions, and that it regulates germline differentiation via germline dpERK signals. Furthermore, we performed a transcriptome analysis of CG6015-mediated regulatory network using RNA sequencing (RNA-seq) and revealed the role of EGFR signaling in spermatogonia TA-divisions in Drosophila testes.

\section{Results}

\section{Spermatogonia derived CG6015 was necessary for cell} differentiation and elongated spermatozoon development

To investigate the role of CG6015 in spermatogonia in Drosophila testes, we employed the upstream activating sequence (UAS)/Gal4 system to manipulate the levels of CG6015 during spermatogonia TA-divisions, which was driven by Bam-Gal4. Quantitative real-time reverse transcription PCR (qRT-PCR) was used to verify the knockdown efficiency and reduced relative mRNA level of CG6015 in CG6015 RNAi testes (Fig. 1a). DNA staining could mark the early-stage nuclei at the apex of the testis and the nuclei of clusters of elongated spermatozoa at the tail of the testis ${ }^{23,24}$. In this study, we found that earlystage cells accumulated and the distance of deeply stained nuclei increased at the apex of Bam>CG6015 RNAi testes, indicating that the differentiation process was inhibited after reducing the CG6015 level in spermatogonia (Fig. $1 \mathrm{~b}-\mathrm{c})$. Previous reports have shown that the Bam protein, which acts as a key differentiation factor, is essential for germ cell differentiation in both testes and ovaries in Drosophila ${ }^{25-28} . \Delta 86 /+$, a heterozygous bam $^{\Delta 86}$ null allele $^{29}$, has been used to enhance germ cell differentiation phenotype in Drosophila testes ${ }^{25}$. Here, differentiation defects were further enhanced by heterozygous mutation of bam $(\Delta 86 /+)$ in Bam>CG6015 RNAi testes (Fig. 1b-c).

Meanwhile, we also observed that the structure of clusters of elongated spermatids was loose and the number of nuclei of the clusters of elongated spermatids was reduced at the tail of CG6015 RNAi testis when compared with the controls (Fig. 1d-e). Our results demonstrated that the number of clusters of elongated spermatozoa was dramatically decreased in Bam>CG6015 RNAi; $\Delta 86 /+$ testes (Fig. $1 \mathrm{~d}-\mathrm{e}$ ). Taken together, our data indicated that CG6015 participates in differentiation and affects elongated spermatozoon formation in Drosophila testes.

\section{CG6015 was required for spermatogonia TA-divisions}

To further explore the roles of CG6015 during germline differentiation, we analyzed morphological changes of fusomes using immunofluorescence staining. Fluorescence tomography imaging has shown that fusomes, which are connections and special structures among germ cells, undergo dynamic changes from a punctate to a branched morphology during germline differentiation $^{30,31}$. In this study, we observed that undifferentiated germ cells, which were marked by DNA and Vasa, accumulated in Bam>CG6015 RNAi testes (Fig. 2a-c). Moreover, the phenotype of germline differentiation defects could be enhanced in the background of heterozygous mutation of bam in Bam>CG6015 RNAi testes (Fig. 2a-d). Meanwhile, the number of punctate fusomes increased and the number of branched fusomes decreased in Bam>CG6015 RNAi and Bam>CG6015 RNAi; $\Delta 86 /+$ testes when compared with control testes (Fig. 2e-f).

To further analyze the differentiation situation of germ cells, we examined Bam-green fluorescent protein (GFP) signals, a recombination protein marks endogenous Bam expression level ${ }^{32}$, in $\Delta 86 /+$, Bam $>C G 6015 \mathrm{RNAi}$, and Bam $>C G 6015$ RNAi; $\Delta 86 /+$ testes. In $\Delta 86 /+$ testes, a 


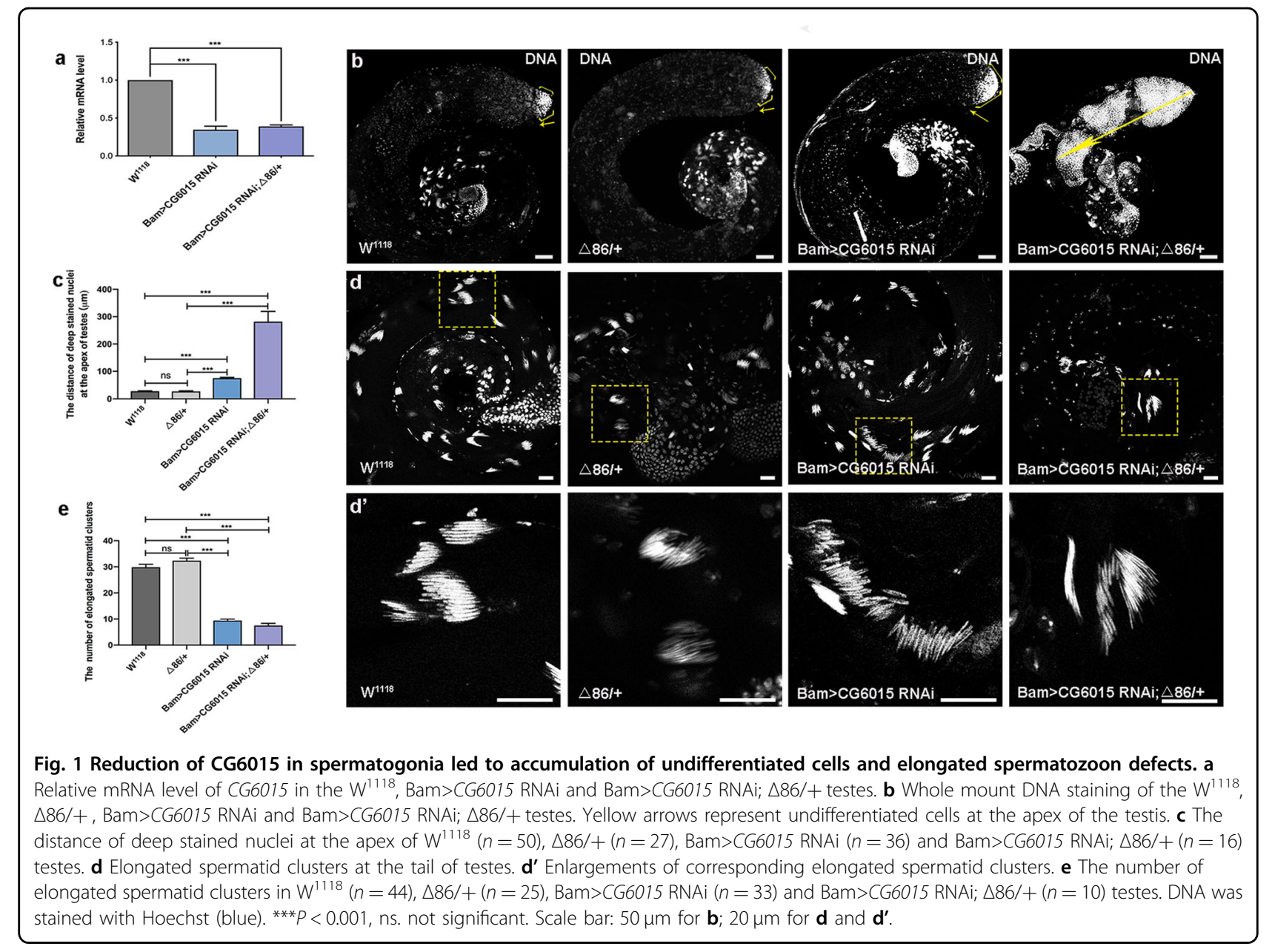

high level of Bam-GFP signals was detected among differentiated spermatogonia (Figs. $2 \mathrm{~g}$ and $2 \mathrm{j}$ ) as previous reported $^{25}$. However, the Bam-GFP signal could be almost undetectable in Bam>CG6015 RNAi or Bam>CG6015 RNAi; $\Delta 86 /+$ testes, which indicated that the germline differentiation process was inhibited by the loss of CG6015 in spermatogonia (Fig. 2g-j).

\section{Spermatogonia derived CG6015 non-autonomously contributed to the cyst cell differentiation}

Previous studies have shown that GSC regulators could maintain the testicular structure and cyst cell survival ${ }^{22}$. We next investigated the maintenance of cyst cells using cyst cell markers (Zfh1 and Eya) under conditions of loss of CG6015 in spermatogonia. In control testes, Zfh1 could label cyst stem cells (CySCs) and the early stage of cyst cells, while Eya was the marker of mature cyst cells ${ }^{31,33}$. The decay of Zfh1 is necessary for promoting the differentiation of cyst cell lineage ${ }^{33}$. Unexpectedly, in both Bam>CG6015 RNAi and Bam>CG6015 RNAi; $\Delta 86 /+$ testes, we found that the number of early-stage cyst cells and mature cyst cells were significantly increased when compared with control testes (Supplementary Fig. S1a-f). More importantly, the number of Eya/Zfh1 double positive cells was also obviously increased in both Bam>CG6015 RNAi and Bam>CG6015 RNAi; $\triangle 86 /+$ testes (Supplementary Fig. S1g). These data indicated that reduction of CG6015 in spermatogonia could also disrupt the differentiation of cyst cells via non-autonomous effects.

\section{CG6015 RNAi in spermatogonia caused abnormal proliferation}

Early stages of germ cells could be maintained and controlled by hub signals ${ }^{34}$. CySCs play instructive roles for the proper onset of the germline differentiation, and loss of CySCs would lead to accumulation of GSC-like cells and failure to enter the TA process in Drosophila testes $^{35}$. Although CySCs and mature cyst cells provide differentiation microenvironments, we found that reduction of CG6015 in spermatogonia directly led to differentiation defects without normal hub signals, and undifferentiated GSC-like cells, which were far away from hub cells, also acquired self-proliferation ability 

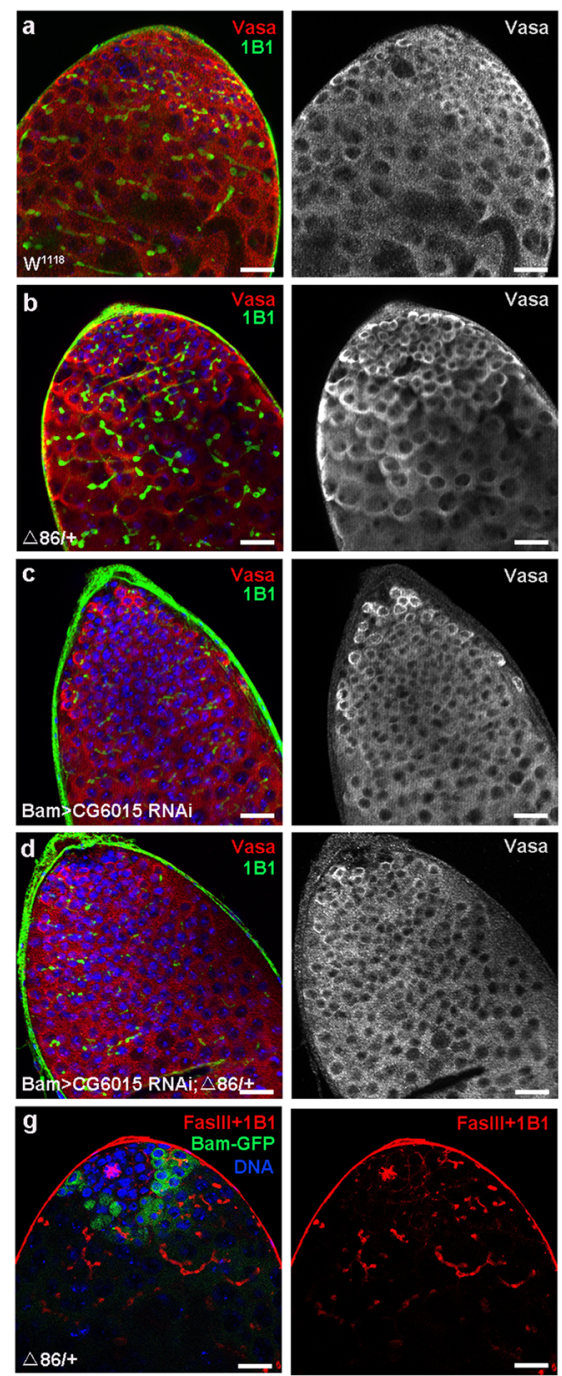

h
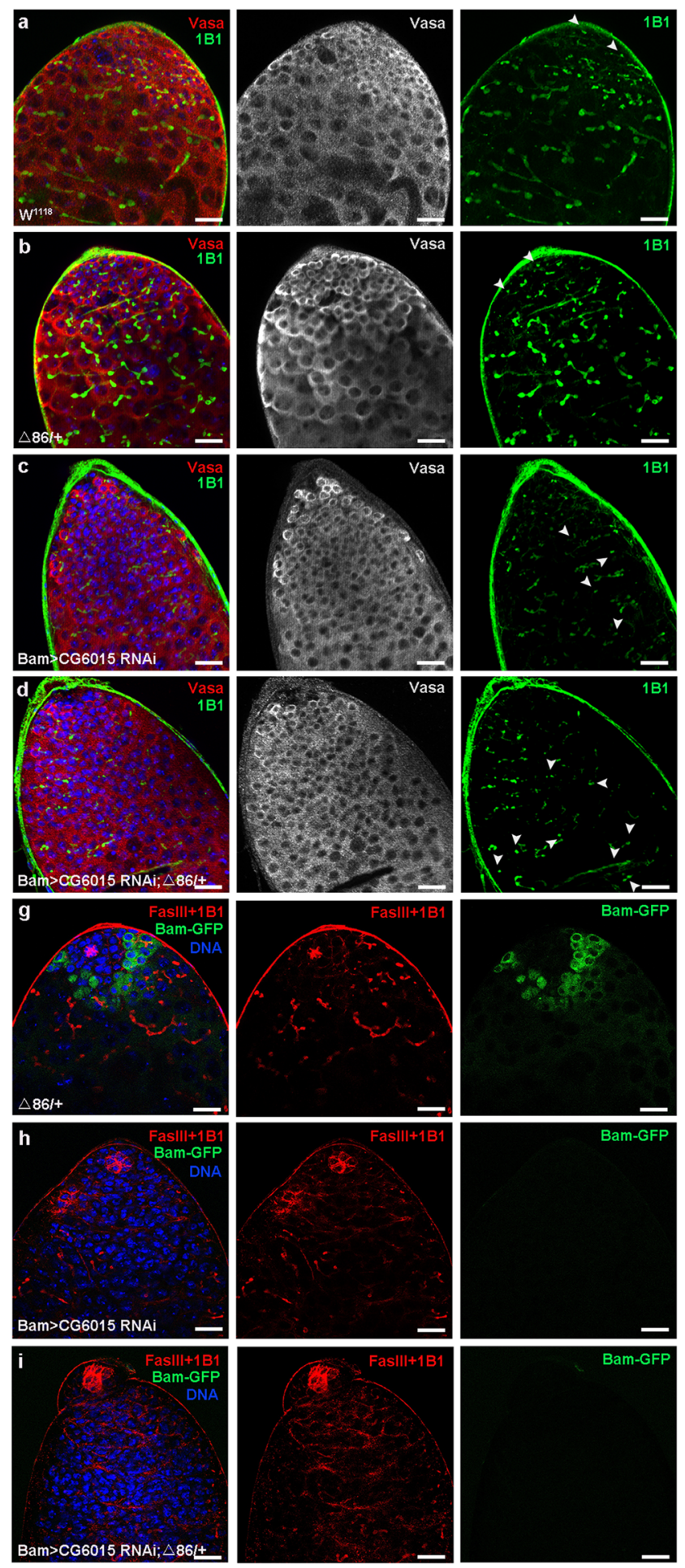

Fig. 2 Reduction of CG6015 in spermatogonia restrained germline differentiation. a-d Immunostaining of Vasa (red) and 1B1 (green) at the apex of $W^{1118}, \Delta 86 /+$, Bam>CG6015 RNAi and Bam>CG6015 RNAi; $\triangle 86 /+$ testes. e The number of punctate fusomes (also defined as 'unbranched fusomes' among germ cells) at the apex of $\mathrm{W}^{1118}(n=13), \Delta 86 /+(n=12), \operatorname{Bam}>C G 6015 \mathrm{RNAi}(n=11)$ and Bam>CG6015 RNAi; $\triangle 86 /+(n=13)$ testes f The number of branched fusomes at the apex of $\mathrm{W}^{1118}(n=13), \triangle 86 /+(n=12), \operatorname{Bam}>C G 6015 \operatorname{RNAi}(n=11)$ and Bam>CG6015 RNAi; $\triangle 86 /+(n=$ 13) testes. g-i Immunostaining of FasIII (red), 1 B1 (red) and Bam-GFP (green) at the apex of $\triangle 86 /+$, Bam>CG6015 RNAi and Bam>CG6015 RNAi; $\triangle 86 /$ + testes. $\mathbf{j}$ Fluorescence intensity of Bam-GFP at the apex of $\triangle 86 /+(n=15), \operatorname{Bam}>C G 6015 \mathrm{RNAi}(n=3)$ and Bam>CG6015 RNAi; $\triangle 86 /+(n=3)$ testes. DNA was stained with Hoechst (blue). ${ }^{* *} P<0.001$, ns. not significant. Scale bars: $20 \mu \mathrm{m}$. 

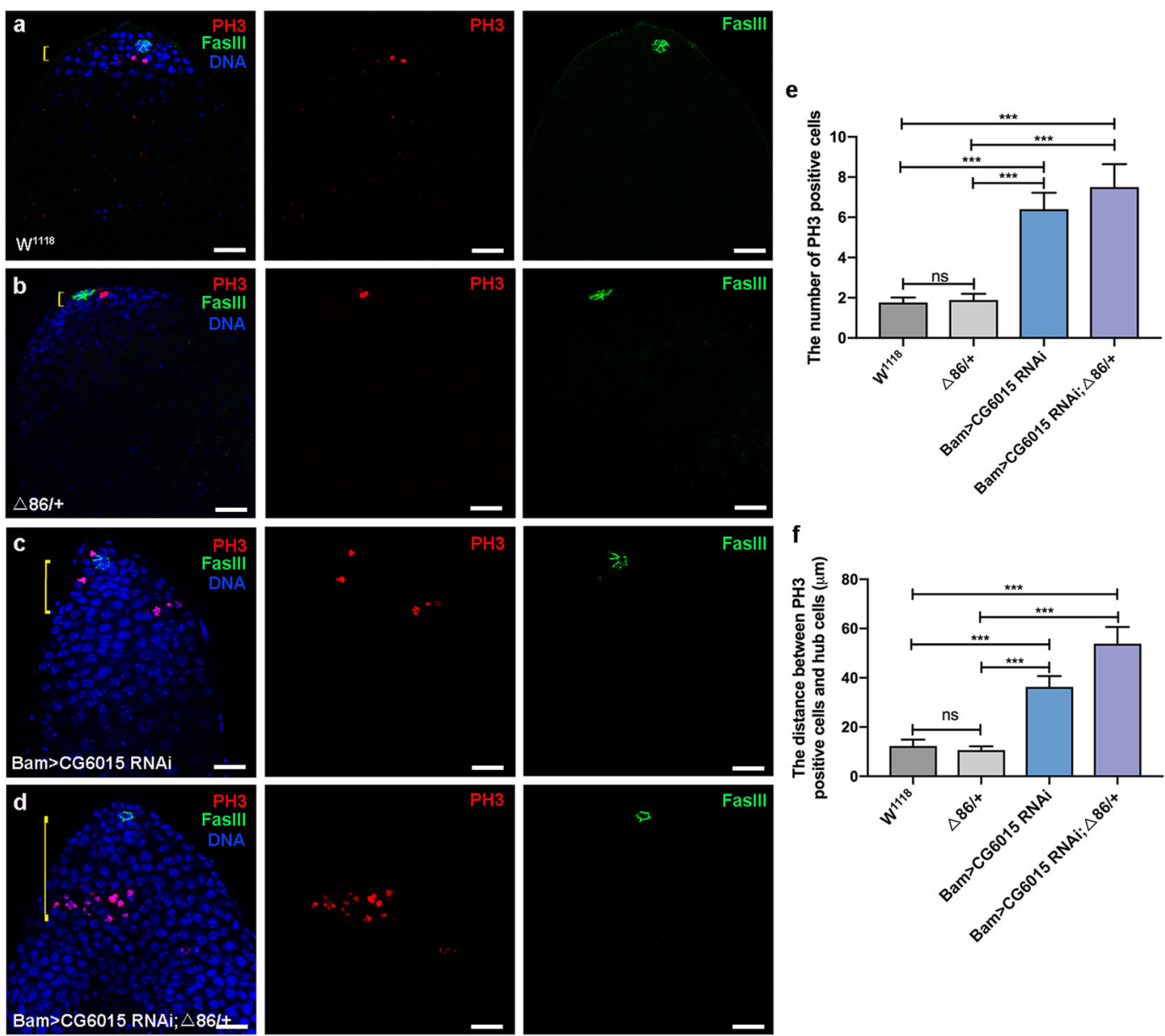

Fig. 3 Reduction of CG6015 in spermatogonia increased proliferation in Drosophila testes. a-d Immunostaining of PH3 (red) and Faslll (green) at the apex of $W^{1118}, \Delta 86 /+$, Bam>CG6015 RNAi and Bam>CG6015 RNAi; $\triangle 86 /+$ testes. DNA was stained with Hoechst (blue). Yellow lines represented the distance between PH3 positive cells and hub cells. e The number of PH3 positive cells in $\mathrm{W}^{1118}(n=12), \triangle 86 /+(n=9), \mathrm{Bam}>C \mathrm{CG} 6015$ $\operatorname{RNAi}(n=10)$ and Bam>CG6015 RNAi; $\triangle 86 /+(n=10)$ testes. f The distance between PH3 positive cells and hub cells in $\mathrm{W}^{1118}(n=12), \Delta 86 /+(n=9)$, Bam>CG6015 RNAi $(n=11)$ and Bam>CG6015 RNAi; $\triangle 86 /+(n=10)$ testes. ${ }^{* *} P<0.001$, ns. not significant. Scale bars: $20 \mu \mathrm{m}$.

(Fig. 3a-d). Moreover, the number of PH3-positive cells and the distance between PH3-positive and hub cells were increased in both Bam>CG6015 RNAi and Bam>CG6015 RNAi; $\Delta 86 /+$ testes when compared with those of control testes (Fig. 3e-f).

\section{Transcriptome analysis of CG6015 mediated regulatory network}

To gain better understandings of the regulatory network mediated by CG6015 in testes, we next carried out RNAseq analysis of control, Bam>CG6015 RNAi, and Bam>CG6015 RNAi; $\triangle 86 /+$ testes. In total (Supplementary Tables S1-S2), 42895 isoforms and 18456 genes were detected in the transcriptional profiles, and differentially expressed isoforms and genes were further analyzed [false discovery rate $(\mathrm{FDR})<0.05 ; \log _{2}$ fold-change $(\mathrm{FC})>1$ or $\log _{2} \mathrm{FC}<-1$ ]. Among them (Fig. 4a and Supplementary
Tables S3-S4), we identified 15384 isoforms (5414 upregulated isoforms and 9970 downregulated isoforms) corresponding to 8530 differentially expressed genes (2734 upregulated genes and 5796 downregulated genes) between the control and Bam>CG6015 RNAi testes. Moreover, we analyzed differentially expressed isoforms and genes between the control and Bam>CG6015 RNAi; $\Delta 86 /+$ testes, and found 16068 isoforms (4634 upregulated isoforms and 11434 downregulated isoforms) corresponding to 8989 differentially expressed genes (2494 upregulated genes and 6495 downregulated genes).

Furthermore, volcano plots and MA plot views revealed distinct differences between the groups (control vs. Bam>CG6015 RNAi; or Control vs. Bam>CG6015 RNAi; $\Delta 86 /+)$ (Fig. $4 \mathrm{~b}-\mathrm{c}$ and Supplementary Fig. S2a-b). Importantly, circular heatmaps displayed the integrity distributions of the expression values and fold changes for 


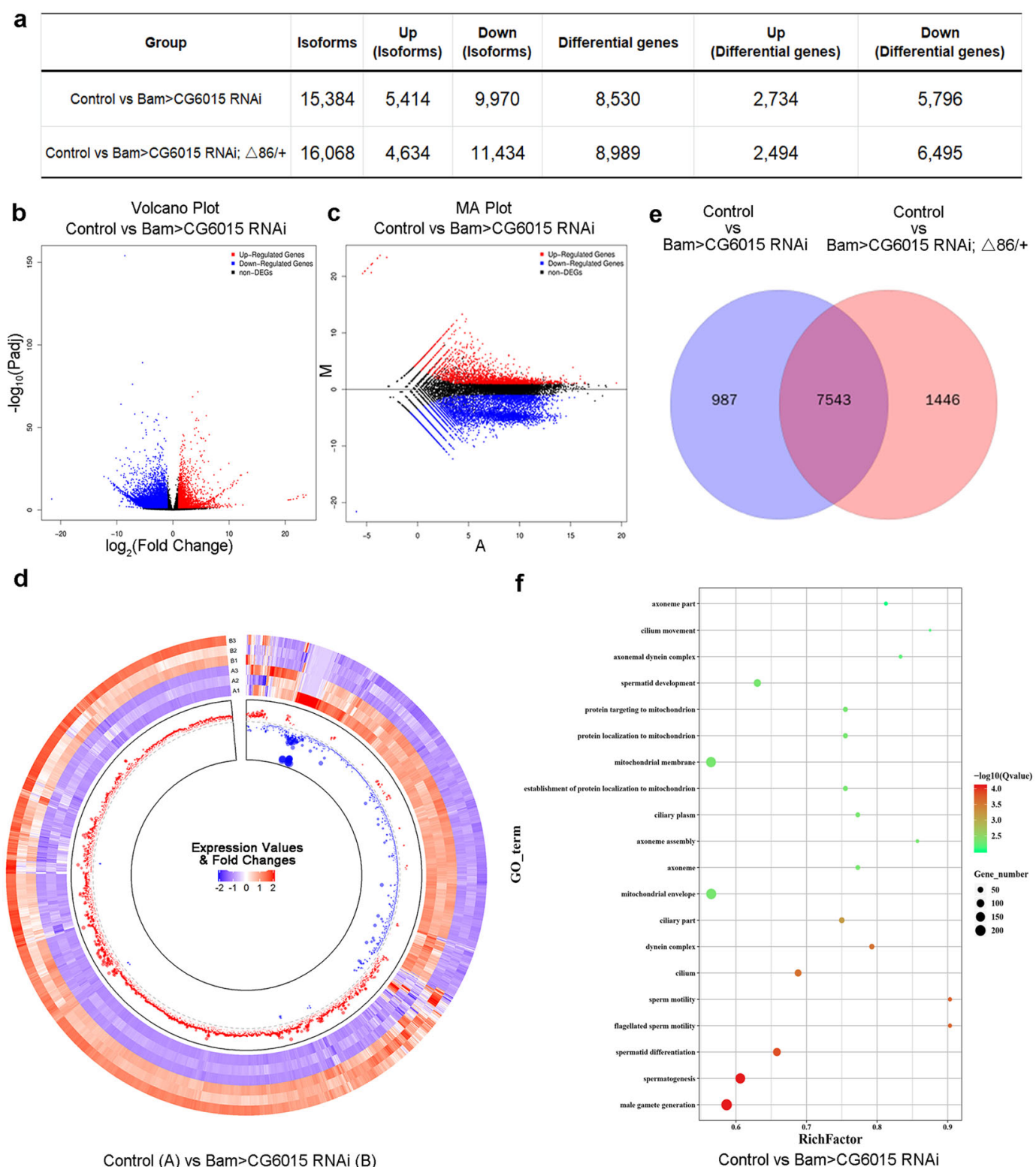

Fig. 4 Reduction of CG6015 in spermatogonia induced transcript alterations as assessed using RNA-seq analysis. a Number of isoforms and genes identified by the RNA-seq in testes. $\mathbf{b}$ Volcano plots based on - $\log _{10} P a d j$ and $\log _{2} F C$ from the comparison of the control $\left(\mathrm{W}^{1118}\right)$ and Bam>CG6015 RNAi groups. c MA plots from the comparison of the control $\left(W^{1118}\right)$ and Bam>CG6015 RNAi groups. $\mathbf{d}$ Circular heatmap of differentially expressed genes from the comparison of the control $\left(W^{1118}\right)$ and Bam>CG6015 RNAi groups. The outer and inner tracks represent the expression values and fold changes, respectively. For both the expression value and fold change, blue and red scales represented low and high expression correspondingly. e Venn diagrams comparing the sets of differentially expressed genes. $\mathbf{f} \mathrm{GO}$ analysis of the sets of differentially expressed genes from the comparison of the control $\left(W^{1118}\right)$ and Bam>CG6015 RNAi groups.

the differentially expressed genes in testes (Fig. 4d and Supplementary Fig. S2c). When the altered transcripts were compared among the three groups (control, Bam>CG6015 RNAi, and Bam>CG6015 RNAi; $\Delta 86 /+$ ), 7543 overlaps were identified, leading us to conclude that CG6015-mediated transcriptional regulation was important for male differentiation in testes (Fig. 4e).
To further analyze the biological events, gene ontology (GO) analysis was performed for the differentially expressed genes induced by spermatogonia CG6015 RNAi. Interestingly, CG6015 RNAi-mediated differentially expressed genes were mainly enriched in male gamete generation, spermatogenesis, spermatid differentiation, sperm motility, axoneme assembly, and protein 
localization to mitochondrion (Fig. 4f and Supplementary Fig. S2d). Moreover, examination of the downregulated differentially expressed genes revealed enrichments that were involved in spermatogenesis and spermatid differentiation, while the upregulated differentially expressed genes mainly participated in metabolic process (Supplementary Fig. S3). Taken together, the transcriptional profile analysis demonstrated that spermatogonia-derived CG6015 regulated spermatogenesis, spermatid differentiation, and metabolic processes.

\section{CG6015-mediated signaling pathways during spermatogonia TA-divisions}

To explore key regulators and identify regulatory signaling pathways during spermatogonia differentiation, we performed pathway enrichment using Gene Set Enrichment Analysis (GSEA) of the global expression transcriptome profiles (Supplementary Tables S5-S6). Interestingly, we identified that the EGFR signaling pathway was enriched in the CG6015-mediated regulatory network in testes (Fig. 5a, b and Supplementary Fig. S4a-b). Moreover, box plot analysis of all identified genes and differentially expressed genes revealed that EGFR signaling was activated in CG6015 RNAi or CG6015 RNAi; $\Delta 86 /+$ testes when compared with that in the controls (Fig. 5c, d and Supplementary Fig. S4c, d). Protein-protein interaction analysis further identified key modules of the EGFR signaling pathway with expression values and enrichments during spermatogonia TAdivisions (Fig. 5e and Supplementary Fig. S4e). We next verified the relative mRNA levels of representative regulators for the EGFR signaling pathway, and found that tumbleweed (tum), GTPase regulator associated with FAK (Graf), and aveugle (ave) were downregulated (Fig. 5f) while rau, kekkon 1 (kek1), and Ras oncogene at 85D (Ras85D) were upregulated (Fig. 5g) in Bam>CG6015 RNAi and Bam>CG6015 RNAi; $\Delta 86 /+$ testes when compared with those in the controls. This qRT-PCR verification was basically consistent with transcriptome profiling data. These data we provided clues to the relationship between CG6015 and the EGFR signaling pathway during spermatogonia TA-divisions in Drosophila testes.

\section{Spermatogonia derived CG6015 was essential for inhibiting germline dpERK}

To exam the effect of the EGFR signaling pathway in spermatogonia in Drosophila testes, we next stained with dpERK, which is a positive effector of the EGFR signaling pathway and was mainly expressed in $\mathrm{CySCs}^{36}$. Germline dpERK signals were upregulated in spermatogonia in Bam>CG6015 RNAi testes, and this phenotype was dramatically enhanced in Bam>CG6015 RNAi; $\triangle 86 /+$ testes (Fig. $6 \mathrm{a}-\mathrm{c}$ ). Furthermore, co-staining of dpERK and 1B1 demonstrated that a series of germline dpERK signals, mediated by CG6015 RNAi, were tightly integrated through fusomes in testes, indicating that these germ cells originated from the same GSCs (Fig. 6d-g). To further confirm the consistency in testes, we quantified the number of fusomes with germline dpERK signals, and discovered that there were $0.8333 \pm 0.2748$ relevant fusomes in Bam>CG6015 RNAi testes $(n=6)$ and $7.286 \pm$ 1.104 relevant fusomes in Bam $>C G 6015$ RNAi; $\Delta 86 /+$ testes $(n=14)$ when compared with control testes (no relevant fusome, $n=12$ ). Therefore, we speculated that CG6015 RNAi in spermatogonia led to differentiation defects and subsequently activated dpERK signaling in GSC-like cells.

\section{Downstream of raf1 (Dsor1) was required for spermatogonia differentiation and regulated germline dpERK in Drosophila testes}

Dsor1 is also a critical downstream mediator of the EGFR signaling pathway, and could phosphorylate ERK ${ }^{37}$. We next investigated the function of Dsor1 in spermatogonia in Drosophila testes. The results showed that downregulation of Dsor1 driven by Bam-Gal4 led to germ cell differentiation defects and induced GSC-like cysts (Fig. 7a, b). Moreover, germline differentiation defects were enhanced by heterozygous mutation of $\operatorname{bam}(\Delta 86 /+)$ in Bam $>$ Dsor1 RNAi testes (Figs. 7a and c). Meanwhile, the number of punctate fusomes was increased in both Bam $>$ Dsor1 RNAi and Bam $>$ Dsor1 RNAi; $\Delta 86 /+$ testes (Fig. 7d). Furthermore, the number of PH3-positive cells and the distance between PH3-positive cells and hub cells were dramatically increased in Bam $>$ Dsor 1 RNAi and Bam $>$ Dsor 1 RNAi; $\Delta 86 /+$ testes, when compared with those in control testes (Fig. 7e-i). We next stained with Vasa and dpERK to estimate germline dpERK signals in Dsor1 RNAi testes. Notably, reduction of Dsor1 in spermatogonia activated germline dpERK signals in $B a m>D$ sor1 RNAi and Bam $>$ Dsor1 RNAi; $\Delta 86 /+$ testes (Supplementary Fig. S5). Our data demonstrated that Dsor1 mimicked the phenotype of CG6015 in spermatogonia and regulated the abundance of dpERK in Drosophila testes.

\section{Discussion}

Spermatogonia TA-divisions control germline homeostasis and instruct the precise transition from proliferation to meiotic differentiation ${ }^{5}$. Bam, a key differentiation factor, is specifically expressed among differentiated spermatogonia, and ectopic expression of Bam promoted germline differentiation $^{38}$. Furthermore, evidence showed that three RNA binding proteins (Tut, Bam, and Bgcn) formed a physical and functional complex to regulate spermatogonia TA-divisions in Drosophila, and were necessary to repress mei-P26 to promote GSC differentiation ${ }^{26}$. 

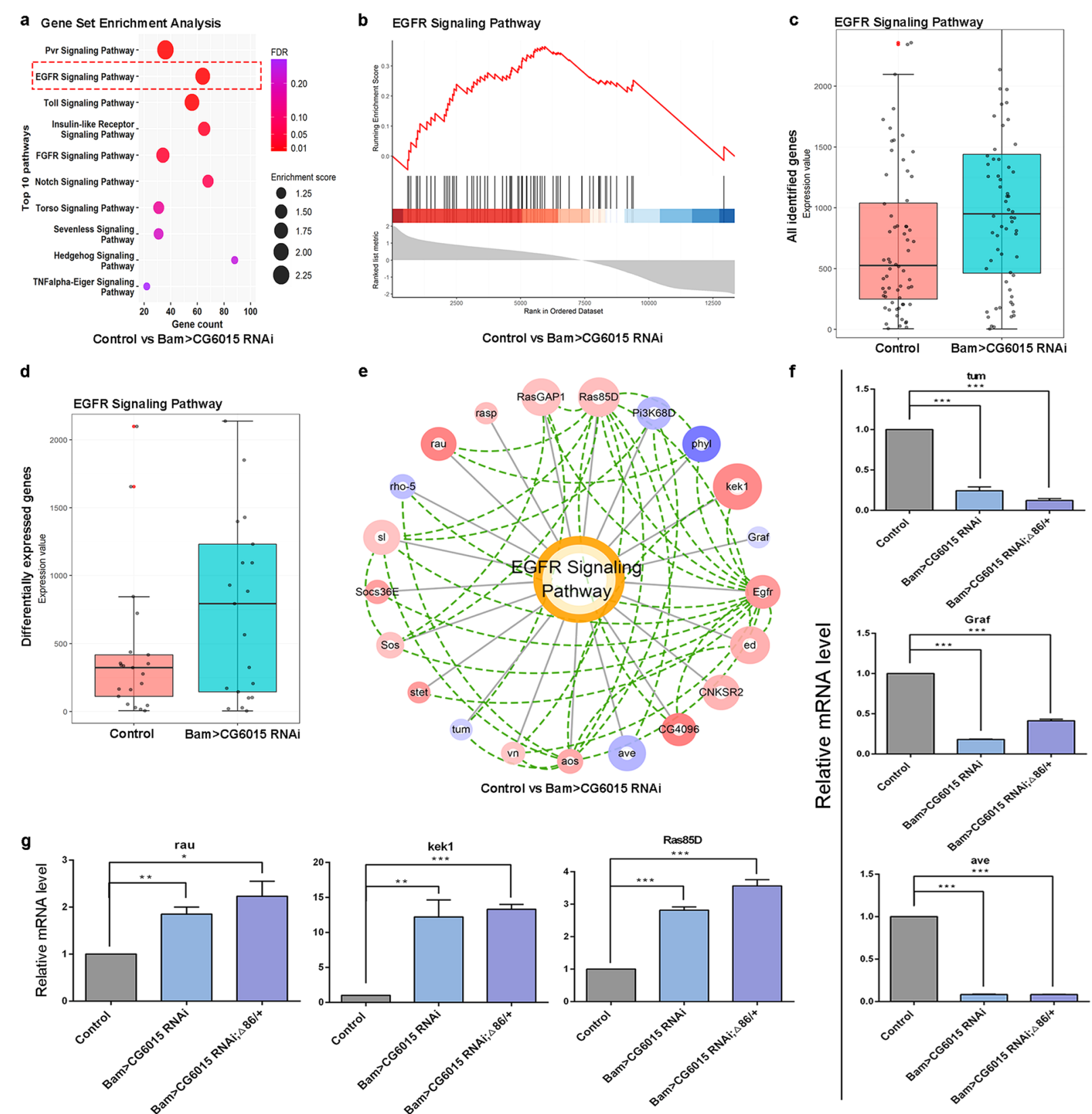

Fig. 5 Inferred signaling pathways and CG6015-mediated regulators during spermatogonia TA-divisions. a Dot plots of the top 10 enriched pathways by GSEA from the comparisons of the control vs. Bam>CG6015 RNAi testes. Color scale and dot size represent FDR value and enrichment score, respectively. $\mathbf{b}$ Enrichment plot for the EGFR pathway from the comparisons of the control vs. Bam>CG6015 RNAi testes. Enrichment plot showing the distribution of the enrichment score, leading genes, and ranked list metric. c Box plots of EGFR signaling for all identified genes from the comparisons of the control vs. Bam>CG6015 RNAi testes. $\mathbf{d}$ Box plots of EGFR signaling for differentially expressed genes from the comparisons of the control vs. Bam>CG6015 RNAi testes. e Expression-interaction network of the EGFR signaling pathway from the comparisons of the control vs. Bam>CG6015 RNAi testes. Blue and red scales represented low and high expression, respectively, while the circle size is proportional to the statistical significance (-logFDR). Green lines represented protein interactions. $\mathbf{f}$ Relative mRNA level of representative down-regulated genes in the control, Bam>CG6015 RNAi and Bam>CG6015 RNAi; $\triangle 86 /+$ testes. g Relative mRNA levels of representative upregulated genes in the control, Bam $>C G 6015$ RNAi and Bam>CG6015 RNAi; $\triangle 86 /+$ testes. ${ }^{*} P<0.05$, ${ }^{* *} P<0.01,{ }^{* *} P<0.001$.

In Drosophila testes, CySCs and hub cells form niche signals and provide an instructive microenvironment for GSC self-renewal ${ }^{39}$. Meanwhile, CySCs could provide somatic signals which trigger GSC differentiation, and deficiency of CySCs and cyst cells resulted in failure to exit the TA-divisions and inhibited germline 

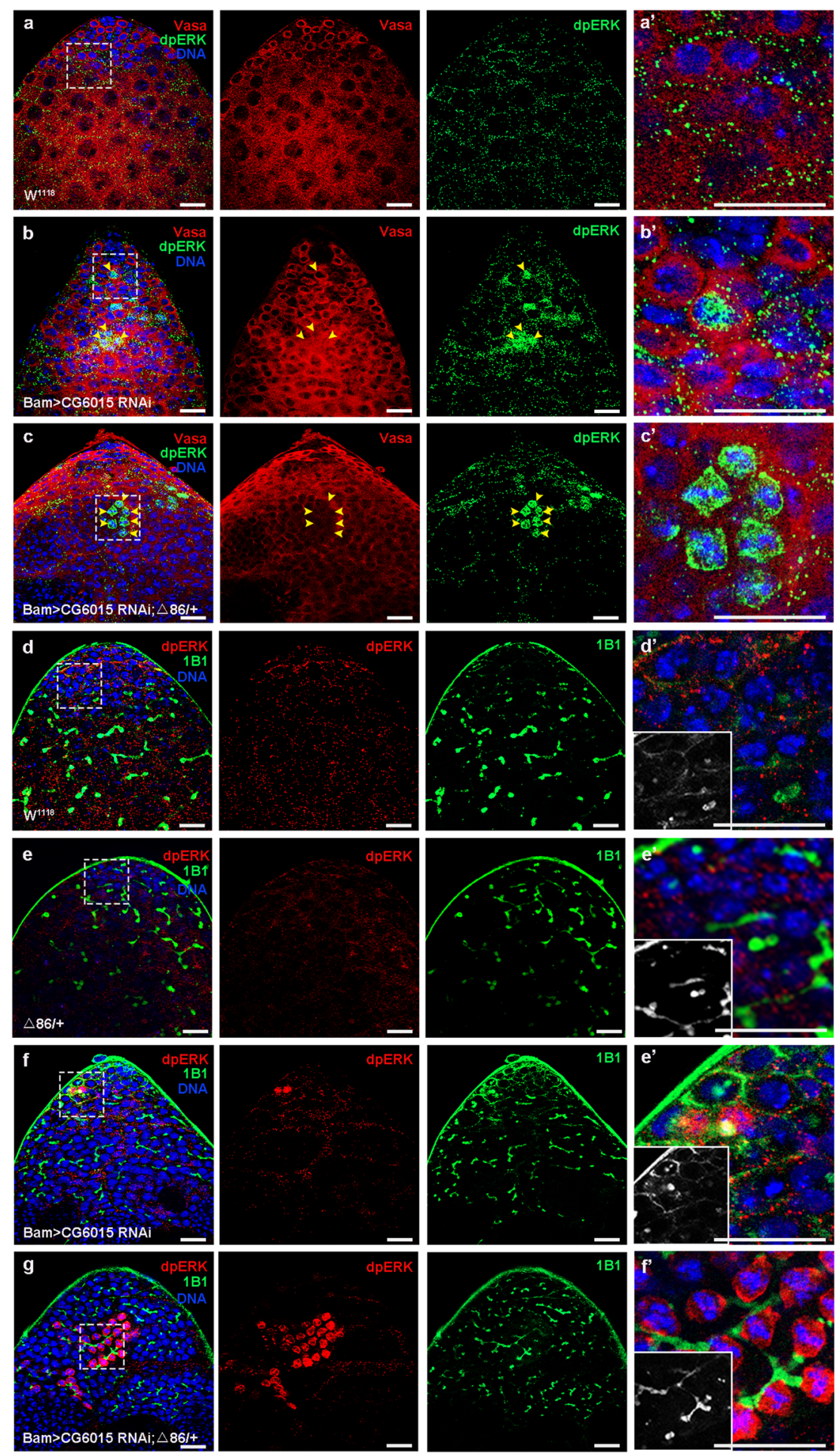

Fig. 6 Reduction of CG6015 in spermatogonia upregulated dpERK in GSC-like cells. a-c Immunostaining of dpERK (green) and Vasa (red) at the apex of $W^{1118}$, Bam>CG6015 RNAi, and Bam>CG6015 RNAi; $\triangle 86 /+$ testes. Representative germline dpERK signals are shown by using yellow arrowheads. $\mathbf{a}^{\prime}-\mathbf{c}^{\prime}$ Enlargements of the immunostaining of dpERK and Vasa signals. $\mathbf{d}-\mathbf{g}$ Immunostaining of dpERK (red) and $1 \mathrm{~B} 1$ (green) at the apex of $W^{1118}, \Delta 86 /+$, Bam>CG6015 RNAi and Bam>CG6015 RNAi; $\triangle 86 /+$ testes. $\mathbf{d}^{\prime}-\mathbf{g}^{\prime}$ Enlargements of immunostaining of dpERK and $1 B 1$ signals. DNA was stained with Hoechst (blue). Scale bars: $20 \mu \mathrm{m}$. 

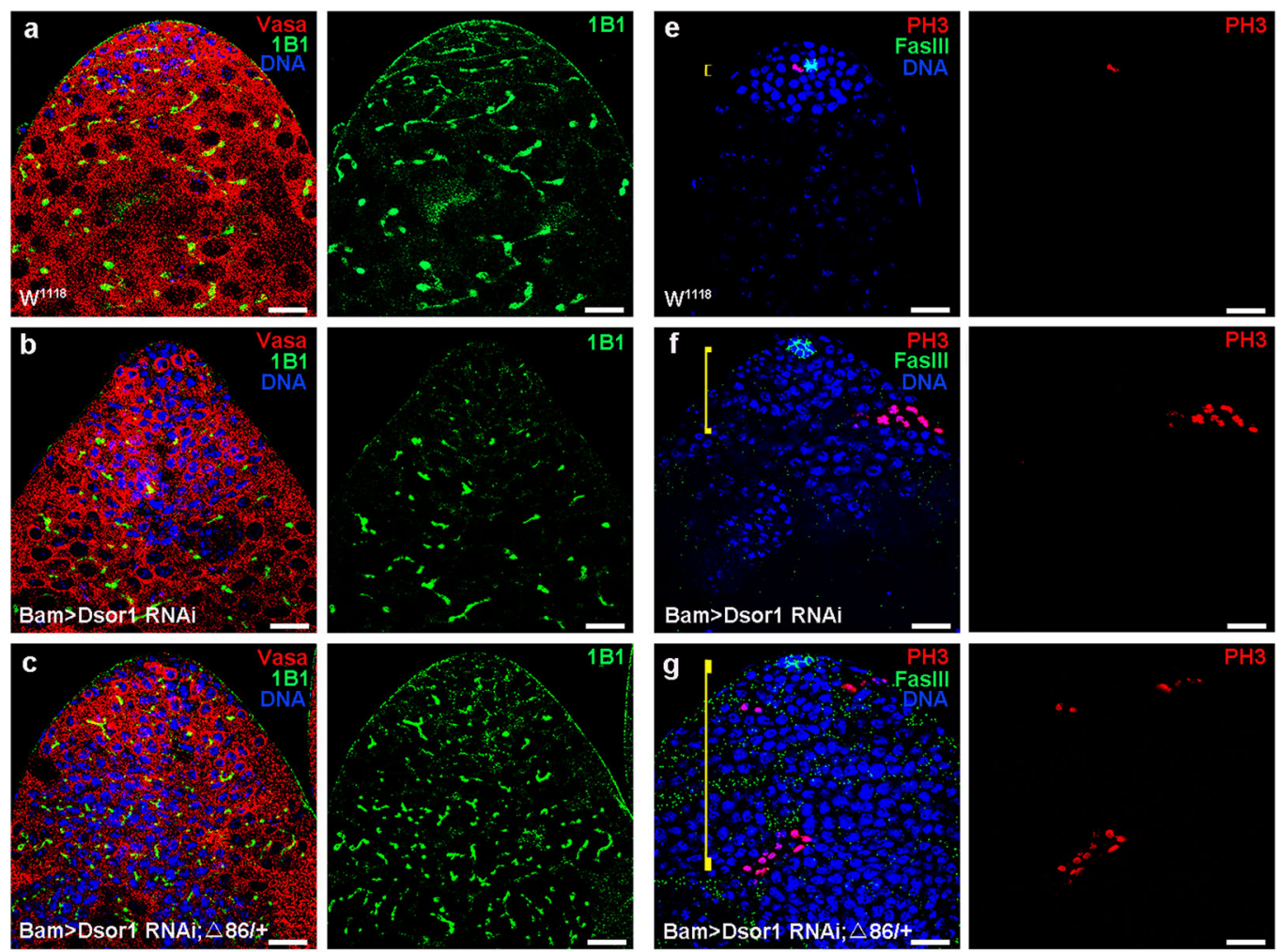

d

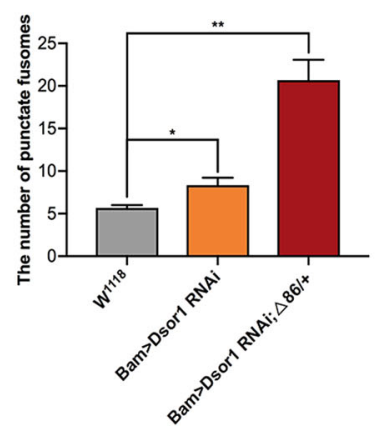

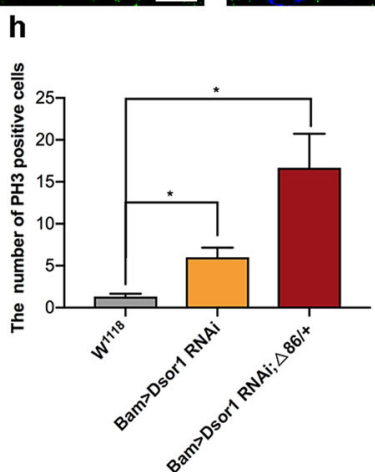

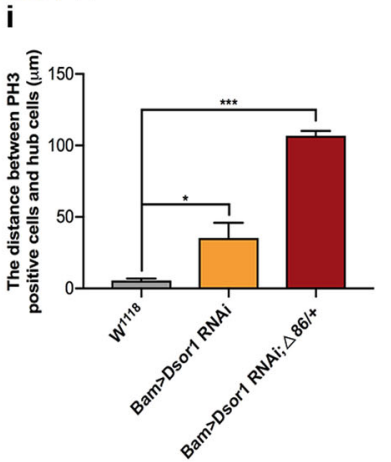

Fig. 7 Downregulation of Dsor1 in spermatogonia resulted in germline differentiation defects. a-c Immunostaining of Vasa (red) and 1B1 (green) at the apex of $W^{1118}$, Bam $>$ Dsor1 RNAi, and Bam>Dsor1 RNAi; $\triangle 86 /+$ testes. $\mathbf{d}$ The number of punctate fusomes in $W^{1118}(n=3)$, Bam>Dsor1 RNAi $(n=3)$, and Bam>Dsor1 RNAi; $\triangle 86 /+(n=3)$ testes. e-g Immunostaining of PH3 (red) and Faslll (green) at the apex of W ${ }^{1118}$, Bam>Dsor1 RNAi, and Bam>Dsor1 RNAi; $\triangle 86 /+$ testes. Yellow lines represent the distance between PH3-positive cells and hub cells. $\mathbf{h}$ The number of PH3-positive cells in $W^{1118}(n=3), \operatorname{Bam}>$ Dsor1 RNAi $(n=3)$, and Bam>Dsor1 RNAi; $\triangle 86 /+(n=3)$ testes. $\mathbf{i}$ The distance between PH3-positive cells and hub cells in $\mathrm{W}^{1118}(n=3)$, Bam>Dsor1 RNAi $(n=3)$, and Bam>Dsor1 RNAi; $\triangle 86 /+(n=3)$ testes. DNA was stained with Hoechst (blue). ${ }^{*} P<0.05,{ }^{* *} P<0.01,{ }^{* * *} P<$ 0.001. Scale bars: $20 \mu \mathrm{m}$.

differentiation ${ }^{35}$. In contrast to the niche signals, we investigated roles of CG6015 during spermatogonia TAdivisions, and found that CG6015 was essential for germline differentiation via cell autonomous effects in spermatogonia. Loss of function of CG6015 in spermatogonia led to extra accumulation of undifferentiated germ cells that could not differentiate into gametes. We also explored the regulatory network using RNA-seq to clarify the potential mechanisms of EGFR signaling in CG6015-mediated differentiation defects.

Elaborate modifications of the spermatid components are key steps for spermiogenesis in Drosophila. GSCs divide into spermatogonia and undergo four rounds of mitosis, producing 16 spermatocytes. Then, they enter into meiosis and result in 64 spermatids which were connected by cytoplasmic bridges and encapsulated by 
two somatic cyst cells ${ }^{40}$. After meiosis within a cyst, the nuclei of 64 elongated spermatozoa are condensed into a sperm bundle. Via spermatid individualization, mature swimming sperm are finally released ${ }^{41}$. Actually, mitochondria also play critical roles throughout the whole process of spermiogenesis. In Drosophila, mitochondria are distributed in the cytoplasm during early stage of spermatogenesis. By the end of meiosis, mitochondria gradually aggregate and fuse into a two-part spherical structure, named 'Nebenkern'. During spermatid elongation, the mitochondrial derivative-nebenkern starts to lengthen parallel to the axis at both ends ${ }^{42,43}$. In this study, we discovered that the structure of clusters of elongated spermatids was loose in CG6015 RNAi testis when compared with the controls, indicating that CG6015 was required to keep spermatids bundled tightly. Moreover, the number of elongated spermatid clusters was significantly reduced in CG6015 RNAi testis. Importantly, differentially expressed genes, which were mediated by CG6015 RNAi in Drosophila testes, were enriched in protein localization to mitochondrion. These results indicated that spermatogonia-derived CG6015 may function in mitochondrion to maintain spermatids elongation and development. Although mitochondrial behavior during spermiogenesis is fairly well understood, the molecular mechanisms between CG6015 and mitochondrial genes remain further investigations.

The fusomes branches throughout germ cell differentiation within a cyst, synchronizing the behavior of germ cells ${ }^{44}$. Previous study has illustrated the visualization of dot-like fusomes, also named punctate fusomes, at the apical tip of testes connecting GSCs and their daughter GBs, as well as the branched network that strings spermatogonia or spermatocytes together within a cyst $^{45}$. Therefore, the accumulation of punctate fusomes far away from hub cells implies over proliferation or differentiation defects in Drosophila testes. As previous study reported, PH3 staining is used to track mitosis. Usually, 2-, 4-, 8- and 16- cell cysts appeared PH3-labeled signals in wild type testes, indicating that germ cells within a cyst always divide together ${ }^{21}$. Here, PH3-positive cells in CG6015 RNAi testes were dramatically increased, indicating the delayed mitosis mediated by CG6015. Moreover, clusters of PH3-positive cells in CG6015 RNAi testes were far away from hub cells, suggesting that loss of CG6015 in spermatogonia substantially desynchronized the mitosis of germ cells. Altogether, these data displayed the significant roles of CG6015 in regulating germ cells divisions during spermatogonia TA-divisions.

Previous reports showed that downstream effectors of EGFR signaling function through cellular extensions of somatic cyst cells to organize microenvironments for germ cell differentiation in Drosophila testes ${ }^{46}$. Evidence showed that the stet gene, whose encoded protein could catalyze proteolytic cleavage of spi within the Golgi, was required for male fertility and encapsulation of germ cells to promote differentiation via EGFR signaling on somatic cyst cells ${ }^{47}$. The Raf oncogene (Raf), which acts downstream of Ras, activates the MEK/ERK pathway to regulate cell proliferation and differentiation ${ }^{48-50}$. Moreover, Raf deficiency disturbed cyst progenitor cell identity and somatic signaling, and further produced excess undifferentiated germ cells in Drosophila testes ${ }^{51}$. A previous study showed that activation of EGFR signaling in somatic cells repressed Dally, thereby affecting the movement and stability of the Dpp protein, leading to germline differentiation $^{52}$. This evidence indicated that somatic EGFR signaling plays key roles in normal germ cell differentiation and forms crucial associations between soma and germline cells.

dpERK, a key EGFR downstream target, was enriched in cyst cells, but was not expressed in germline cells in Drosophila testes ${ }^{53}$. However, the roles of germline EGFR signaling are rarely studied and discussed, and the mechanism of germline EGFR signaling is unknown. In the present study, we used dpERK to assess the activation of EGFR signaling. We further determined the relationship between CG6015 and EGFR signaling during spermatogonia TA-divisions in Drosophila testes. Interestingly, we observed the existence of germline dpERK signals, and CG6015 mediated germline differentiation defects by directly activating germline dpERK in GSC-like cysts. In addition to the previously reported roles for the formation of CySCs, EGFR signaling also displayed dose- dependent effects on differentiation. Interestingly, a low level of EGFR signaling was required for spermatogonia TA-divisions, and a high level of EGFR signaling promoted germline differentiation in Drosophila testes $^{54}$. Based on the above evidence, we speculated that ectopic production of germline dpERK among undifferentiated GSC-like cells might be an instinctive cellular response to promote differentiation.

In summary, we explored roles of CG6015 during spermatogonia TA-divisions, and revealed the relationship between CG6015 and EGFR signaling in Drosophila testes. Our data strongly suggested that CG6015 regulates germline differentiation via germline dpERK signals. This study extends our understanding of spermatogonia TAdivisions and germline homeostasis, and could be used as the basis to investigate crosstalk between somatic and germline cells.

\section{Materials and methods Fly strains}

All flies were raised on standard cornmeal molasses agar medium at $25^{\circ} \mathrm{C}$ and in a relative humidity of $40-60 \%$. UAS-RNAi transgenic flies were obtained from the TsingHua Fly Center (THFC) and detailed information 
was as follows: UAS-CG6015 RNAi (\#THU1409), UASDsor1 RNAi (\#THU0677). Bam-Gal4; $\Delta 86 /+$ and BamGFP; $\Delta 86 /+$ lines were gifts from DH Chen (Institute of zoology, Chinese Academy of Sciences, Beijing, China).

\section{Fly crosses}

Two to three-day-old flies were used in this study. Male flies of the Bam-Gal4; $\Delta 86 /+$ line were randomly chosen to cross with transgenic UAS-RNAi virgins and raised at $25^{\circ} \mathrm{C}$ until hatching. F1 males with a specific genotype (Bam $>$ RNAi or Bam $>$ RNAi; $\Delta 86 /+$ ) were selected for the further analysis. The $\mathrm{W}^{1118}$ and $\Delta 86 /+$ lines were used as controls.

\section{Quantitative real-time reverse transcription PCR}

Quantitative real-time reverse transcription PCR (qRTPCR) was performed according to the manufacturer's protocol. Total RNA was extracted using TRIzol Reagent (15596026, Invitrogen, Waltham, MA, USA). Reverse transcription was performed using HiScript III 1st Strand cDNA Synthesis Kit (R312-01, Vazyme, Jiangsu, China). TB Green Premix Ex Taq II (RR820, Takara, Shiga, Japan) was used to carry out the qPCR step. The primers used in this study are shown in Supplementary Table S7.

\section{Immunostaining and antibodies}

Fly testes were dissected in $1 \times$ phosphate-buffered saline (PBS), fixed for $30 \mathrm{~min}$ in $4 \%$ paraformaldehyde, washed with $0.3 \%$ PBS-Triton X-100 (PBST) three times, and blocked in $5 \%$ bovine serum albumin for $30 \mathrm{~min}$. Testes were incubated with primary antibodies at room temperature for $1 \mathrm{~h}$. Then, testes were washed three times in $0.3 \%$ PBST and incubated with secondary antibodies at room temperature for $1 \mathrm{~h}$ in the dark. After washing three times again with $0.3 \% \mathrm{PBST}$, testes were stained with Hoechst-33342 $(1.0 \mathrm{mg} / \mathrm{ml}$, C0031, Solarbio, Beijing, China) for $5 \mathrm{~min}$ before mounting. The primary antibodies used were as follows: rat anti-Vasa [Developmental Studies Hybridoma Bank (DSHB), University of Iowa, Dept of Biology, Iowa City, IA, USA; 1:20], mouse anti1B1 (DSHB, 1:50), mouse anti-FasIII (DSHB, 1:50), rat anti-Zfh1 (a gift from Prof. Chao Tong, 1:1000), mouse anti-Eya (DSHB, 1:30), rabbit anti-PH3 [\#53348, Cell Signaling Technology (CST), Danvers, MA, USA; 1:1000], rabbit anti-dpERK (\#4370, CST, 1:200). Secondary antibodies conjugated with A488, Cy3, or A647 (Jackson ImmunoResearch Laboratories, West Grove, PA, USA) were diluted at 1:1000.

\section{Images acquisition and analysis}

The images for Drosophila testes were obtained by microscope and analyzed by Image J software according to usage introductions. For the measurement of distance between hub cells and $\mathrm{PH} 3$ positive cells, Image J was used to draw a line between hub cells and the farthest PH3 positive cells. The measurement results would be given in the form of pixel values. Necessary parameters are required to been set in image $J$ for transforming the measurement data from pixel values to physical dimensions.

\section{Preparation and RNA-Seq}

We used two to three-day-old flies as controls, and Bam>CG6015 RNAi and Bam>CG6015 RNAi; $\Delta 86 /+$ genotypes in this study. Testes from 50 male flies of each genotype were dissected in cold PBS. Total RNA was prepared from the isolated testes using TRIzol (15596026, Invitrogen), following the recommendations of the manufacturer. The concentration and purity of the total RNA were detected using a Nanodrop 2000 instrument (Nanodrop Technologies, Wilmington, DE, USA), and the integrity of the RNA was detected by gel electrophoresis, and the RNA integrity (RIN) values were measured using an Agilent 2100 instrument (Agilent Technologies, Santa Clara, CA, USA). Indexed RNA-Seq libraries were prepared from $800 \mathrm{ng}$ of total RNA using the TruSeq RNA Library Prep Kit v2 (Illumina, San Diego, CA, USA)) following the manufacturer's protocol. This experiment included purification of poly (A) mRNA with oligo-dT magnetic beads, RNA fragmentation, synthesis of doublestranded cDNA using SuperScript II reverse transcriptase (Invitrogen), ligation of indexed Illumina adapters, and amplification using limited-cycle PCR. Sequencing libraries were validated by capillary electrophoresis using a Bioanalyzer 2100 instrument (Agilent Technologies). The DNB (DNA nano ball) was prepared after the libraries were tested for qualification, and then loaded into the sequencing chip. Sequencing was performed using a high-throughput sequencer (MGIseq2000, MGI, Shenzhen, China).

\section{Bioinformatic analysis of RNA-seq data}

Reads were aligned to the Drosophila melanogaster reference genome in NCBI's assembly resource (www. ncbi.nlm.nih.gov/assembly/) using the Bowtie package with Hierarchical Indexing for Spliced Alignment of Transcripts (HISAT) comparison software ${ }^{54}$. The transcript abundances were determined using Fragments Per Kilobase per Million mapped reads (FPKM) values with the RSEM tool ${ }^{55}$. Differentially expressed genes were identified using criteria of a an FDR $<0.05$ and $\log 2 \mathrm{FC}>1$ (or $\log 2 \mathrm{~F}<-1$ ). Volcano plot views were obtained to display visually the distribution of FDR and FC values of differentially expressed genes between two groups. The number of differentially expressed genes among multiple groups was analyzed using Venn diagrams. Gene ontology (GO) enrichment analysis was performed using the hypergeometric distribution for total, downregulated, and 
upregulated differentially expressed genes, and graphs were shown with GO terms, rich factor, gene number, and $-\log 10(\mathrm{Q}$ value). For the circular heatmap, all differentially expressed genes were normalized by the Z-score and clustered using $\mathrm{MeV}$ (version 4.9.0) ${ }^{56}$. The circular heatmap was generated using the circlize package (version 0.4.11) in R software ${ }^{57}$. For GSEA, among all the identified genes, those with extremely low expression values (below 5) were filtered out, and all reserved genes were ranked in descending order according to their degree of change. The reference gene sets (known signaling pathways) were extracted from the gene group annotations in FlyBase ${ }^{58}$. GSEA was performed using the GSEA function embedded in the cluster Profiler package (version 3.18.0) in the $R$ software ${ }^{59}$. An FDR of 0.05 was set as the cutoff for a statistically significant enrichment. Box plots were generated by using the ggplot 2 package in the $\mathrm{R}$ software. The protein-protein interactions were obtained from the STRING database using a medium score ${ }^{60}$. The complex expression-interaction network was generated using Cytoscape (version 3.9.0) ${ }^{61}$.

\section{Statistical analysis for functional experiments}

All the functional experiments conducted in this study were repeated at least three times. The quantitative results were presented as means \pm standard error of the mean (SEM) and evaluated for statistical differences using Student's $t$-test and one-way analysis of variance (ANOVA) using GraphPad software (GraphPad Inc., La Jolla, CA, USA). ${ }^{*} P<0.05 ;{ }^{* * *} P<0.01$; ${ }^{* * * *} P<0.001$.

\section{Acknowledgements}

The authors wish to thank all study participants, research staff, and students who assisted with this work. We would like to thank Chao Tong (Life Sciences Institute, Zhejiang University, Zhejiang, China) and DH Chen (Institute of zoology, Chinese Academy of Sciences, Beijing, China) for sharing reagents and stocks. Funding This work was supported by The National Key Research and Development Program of China (No. 2018YFC1003500), The National Natural Science Foundation of China (81901532, 81901533), Natural Science Foundation of Jiangsu Province (BK20190188), Suzhou Key Laboratory of Male Reproduction Research (SZS201718), Large Instruments Open Foundation of Nantong University (KFJN2157).

\section{Author details}

${ }^{1}$ Institute of Reproductive Medicine, School of Medicine, Nantong University, Nantong, China. ${ }^{2}$ Department of Gynecology, the Affiliated Hospital of Jiangsu University, Jiangsu University, Zhenjiang, China. ${ }^{3}$ State Key Laboratory of Reproductive Medicine, Center for Reproduction and Genetics, Suzhou Municipal Hospital, The Affiliated Suzhou Hospital of Nanjing Medical University, Gusu School, Nanjing Medical University, Suzhou, China

\section{Author contributions}

J.Y., B.Z. and F.S. initiated the project, designed the study, coordinated the experiment and wrote the manuscript. Q.Z., Z.L., Y.W. and Y.F. performed the experiments and provided conceptual inputs for the paper. Q.Z., J.Y., X.W., D.L. and C.S. analyzed the data. All authors read and approved the final manuscript.

Conflict of interest

The authors declare no competing interests.
Ethics statement

This study was approved by the Ethics Committee of Nantong University.

\section{Publisher's note}

Springer Nature remains neutral with regard to jurisdictional claims in published maps and institutional affiliations.

Supplementary information The online version contains supplementary material available at https://doi.org/10.1038/s41419-021-03783-9.

Received: 15 January 2021 Revised: 29 April 2021 Accepted: 30 April 2021 Published online: 14 May 2021

\section{References}

1. Kiger, A. A., White-Cooper, H. \& Fuller, M. T. Somatic support cells restrict germline stem cell self-renewal and promote differentiation. Nature 407 750-754 (2000).

2. Monk, A. C. et al. HOW is required for stem cell maintenance in the Drosophila testis and for the onset of transit-amplifying divisions. Cell Stem Cell 6, 348-360 (2010).

3. Kawase, E., Wong, M. D., Ding, B. C. \& Xie, T. Gbb/Bmp signaling is essential for maintaining germline stem cells and for repressing bam transcription in the Drosophila testis. Development 131, 1365-1375 (2004).

4. Shivdasani, A. A. \& Ingham, P. W. Regulation of stem cell maintenance and transit amplifying cell proliferation by tgf-beta signaling in Drosophila spermatogenesis. Curr. Biol. 13, 2065-2072 (2003).

5. Insco, M. L., Leon, A., Tam, C. H., McKearin, D. M. \& Fuller, M. T. Accumulation of a differentiation regulator specifies transit amplifying division number in an adult stem cell lineage. Proc. Natl Acad. Sci. USA 106, 22311-22316 (2009).

6. Eun, S. H. et al. MicroRNAs downregulate Bag of marbles to ensure proper terminal differentiation in the Drosophila male germline. Development 140, 23-30 (2013).

7. Harden, N. New insights from Drosophila into the regulation of EGFR signaling. Methods Mol. Biol. 1652, 37-42 (2017).

8. Normanno, N. et al. Epidermal growth factor receptor (EGFR) signaling in cancer. Gene 366, 2-16 (2006).

9. Kim, S. Y. et al. Negative regulation of EGFR/MAPK pathway by Pumilio in Drosophila melanogaster. PLoS One 7, e34016 (2012).

10. Fereres, S., Hatori, R., Hatori, M. \& Kornberg, T. B. Cytoneme-mediated signaling essential for tumorigenesis. PLoS Genet 15, e1008415 (2019).

11. Castanieto, A., Johnston, M. J. \& Nystul, T. G. EGFR signaling promotes selfrenewal through the establishment of cell polarity in Drosophila follicle stem cells. Elife 3, e04437 (2014).

12. Nakano, A. \& Takashima, S. LKB1 and AMP-activated protein kinase: regulators of cell polarity. Genes. Cells 17, 737-747 (2012).

13. Johnston, M. J., Bar-Cohen, S., Paroush, Z. \& Nystul, T. G. Phosphorylated Groucho delays differentiation in the follicle stem cell lineage by providing a molecular memory of EGFR signaling in the niche. Development 143, 4631-4642 (2016).

14. Tomer, D., Chippalkatti, R., Mitra, K. \& Rikhy, R. ERK regulates mitochondrial membrane potential in fission deficient Drosophila follicle cells during differentiation. Dev. Biol. 434, 48-62 (2018).

15. Sênos Demarco, R. \& Jones, D. L. FR signaling promotes basal autophagy for lipid homeostasis and somatic stem cell maintenance in the Drosophila testis. Autophagy 16, 1145-1147 (2020).

16. Sênos Demarco, R., Uyemura, B. S. \& Jones, D. L. EGFR signaling stimulates autophagy to regulate stem cell maintenance and lipid homeostasis in the Drosophila testis. Cell Rep. 30, 1101-1116.e5 (2020).

17. Shilo, B. Z. The regulation and functions of MAPK pathways in Drosophila. Methods 68, 151-159 (2014).

18. Hall, E. T. \& Verheyen, E. M. Ras-activated Dsor1 promotes Wht signaling in Drosophila development. J. Cell Sci. 128, 4499-4511 (2015).

19. Courcelles, M. et al. Phosphoproteome dynamics reveal novel ERK1/2 MAP kinase substrates with broad spectrum of functions. Mol. Syst. Biol. 9, 669 (2013).

20. Qian, Y. et al. Ecdysone signaling opposes epidermal growth factor signaling in regulating cyst differentiation in the male gonad of Drosophila melanogaster. Dev. Biol. 394, 217-227 (2014). 
21. Gupta, S., Varshney, B., Chatterjee, S. \& Ray, K. Somatic ERK activation during transit amplification is essential for maintaining the synchrony of germline divisions in Drosophila testis. Open Biol. 8, 180033 (2018).

22. $\mathrm{Yu}, \mathrm{J}$. et al. Protein synthesis and degradation are essential to regulate germline stem cell homeostasis in Drosophila testes. Development $\mathbf{1 4 3}$ 2930-2945 (2016).

23. $Y u$, J. et al. Srlp is crucial for the self-renewal and differentiation of germline stem cells via RpL6 signals in Drosophila testes. Cell Death Dis. 10, 294 (2019).

24. $\mathrm{Yu}, \mathrm{J}$. et al. Small ribonucleoprotein particle protein SmD3 governs the homeostasis of germline stem cells and the crosstalk between the spliceosome and ribosome signals in Drosophila. FASEB J. 33, 8125-8137 (2019).

25. Carbonell, A., Pérez-Montero, S., Climent-Cantó, P., Reina, O. \& Azorín, F. The germline linker histone $\mathrm{dBigH1}$ and the translational regulator Bam form a repressor loop essential for male germ stem cell differentiation. Cell Rep. 21, 3178-3189 (2017)

26. Chen, D. et al. Three RNA binding proteins form a complex to promote differentiation of germline stem cell lineage in Drosophila. PLoS Genet 10, e1004797 (2014)

27. Shen, R., Weng, C., Yu, J. \& Xie, T. elF4A controls germline stem cell self-renewal by directly inhibiting BAM function in the Drosophila ovary. Proc. Natl Acad. Sci. USA 106, 11623-11628 (2009).

28. Tiwari, M. D., Zeitler, D. M., Meister, G. \& Wodarz, A. Molecular profiling of stem cell-like female germ line cells in Drosophila delineates networks important for stemness and differentiation. Biol. Open 8, bio046789 (2019).

29. McKearin, D. \& Ohisthin, B. A role for the Drosophila Bag-of-marbles protein in the differentiation of cystoblasts from germline stem cells. Development $\mathbf{1 2 1}$ 2937-2947 (1995)

30. Chen, W. et al. CG8005 mediates transit-amplifying spermatogonial divisions via oxidative stress in Drosophila testes. Oxid. Med Cell Longev. 2020, 2846727 (2020).

31. Wang, M. et al. RpS13 controls the homeostasis of germline stem cell niche through Rho1-mediated signals in the Drosophila testis. Cell Prolif. 53, e12899 (2020).

32. Chen, D. \& McKearin, D. M. A discrete transcriptional silencer in the bam gene determine asymmetric division of the Drosophila germline stem cell. Development 130, 1159-1170 (2003).

33. Leatherman, J. L. \& Dinardo, S. Zfh-1 controls somatic stem cell self-renewal in the Drosophila testis and nonautonomously influences germline stem cell selfrenewal. Cell Stem Cell 3, 44-54 (2008).

34. de Cuevas, M. \& Matunis, E. L. The stem cell niche: lessons from the Drosophila testis. Development 138, 2861-2869 (2011).

35. Lim, J. G. Y. \& Fuller, M. T. Somatic cell lineage is required for differentiation and not maintenance of germline stem cells in Drosophila testes. Proc. Natl Acad. Sci. USA 109, 18477-18481 (2012).

36. Paul, S. et al. Activation-induced substrate engagement in ERK signaling. Mol. Biol. Cell 31, 235-243 (2020).

37. Roskoski, R. Jr ERK1/2 MAP kinases: structure, function, and regulation. Pharm. Res 66, 105-143 (2012).

38. Sheng, X. R., Brawley, C. M. \& Matunis, E. L. Dedifferentiating spermatogonia outcompete somatic stem cells for niche occupancy in the Drosophila testis. Cell Stem Cell 5, 191-203 (2009).

39. Leatherman, J. L. \& Dinardo, S. Germline self-renewal requires cyst stem cells and stat regulates niche adhesion in Drosophila testes. Nat. Cell Biol. 12 806-811 (2010).
40. Loza-Call, M. A., Petrossian, C. C., Boyle, M. L. \& Jones, D. L. Heterochromatin Protein 1(HP1) inhibits stem cell proliferation induced by ectopic activation of the JAKVSTAT pathway in the Drosophila testis. Exp. Cell Res. 377, 1-9 (2020).

41. Steinhauer, J. et al. Combover interacts with the axonemal component Rsp3 and is required for Drosophila sperm individualization. Development 146, dev179275 (2019).

42. Chen, J., Buchwalter, R. A., Kao, L.-R. \& Megraw, T. A splice variant of Centrosomin converts mitochondria to microtubule-organizing centers. Curr. Biol. 27, 1928-1940 (2017)

43. Laurinyecz, B. et al. Sperm-Leucylaminopeptidases are required for male fertility as structural components of mitochondrial paracrystalline material in Drosophila melanogaster sperm. PLoS Genet. 15, e1007987 (2019).

44. Eikenes, A. H., Brech, A., Stenmark, H. \& Haglund, K. Spatiotemporal control of Cindr at ring canals during incomplete cytokinesis in the Drosophila male germline. Dev. Biol. 377, 9-20 (2013).

45. Kibanov, M. V., Kotov, A. A. \& Olenina, L. V. Multicolor fluorescence imaging of whole-mount Drosophila testes for studying spermatogenesis. Anal. Biochem. 436, 55-64 (2013).

46. Sarkar, A. et al. Antagonistic roles of Rac and Rho in organizing the germ cell microenvironment. Curr. Biol. 17, 1253-1258 (2007).

47. Schulz, C., Wood, C. G., Jones, D. L., Tazuke, S. I. \& Fuller, M. T. Signaling from germ cells mediated by the rhomboid homolog stet organizes encapsulation by somatic support cells. Development 129, 4523-4534 (2002).

48. Lavoie, $\mathrm{H}$. et al. Therrien M. MEK drives BRAF activation through allosteric control of KSR proteins. Nature 554, 549-553 (2018).

49. Jiang, H. \& Edgar, B. A. EGFR signaling regulates the proliferation of Drosophila adult midgut progenitors. Development 136, 483-493 (2009).

50. Kim, M. et al. Inhibition of ERK-MAP kinase signaling by RSK during Drosophila development. EMBO J. 25, 3056-3067 (2006).

51. Tran, J., Brenner, T. J. \& DiNardo, S. Somatic control over the germline stem cell lineage during Drosophila spermatogenesis. Nature 407, 754-757 (2000).

52. Liu, M., Lim, T. M. \& Cai, Y. The Drosophila female germline stem cell lineage acts to spatially restrict DPP function within the niche. Sci. Signal 3, ra57 (2010).

53. Hudson, A. G., Parrott, B. B., Qian, Y. \& Schulz, C. A temporal signature of epidermal growth factor signaling regulates the differentiation of germline cells in testes of Drosophila melanogaster. PLOS ONE 8, e70678 (2013).

54. Kim, D., Langmead, B. \& Salzberg, S. L. HISAT: a fast spliced aligner with low memory requirements. Nat. Methods 12, 357-360 (2015).

55. Li, B. \& Dewey, C. N. RSEM: accurate transcript quantification from RNA-Seq data with or without a reference genome. BMC Bioinformatics 12, 323 (2011).

56. Saeed, A. I. et al. TM4: a free, open-source system for microarray data management and analysis. Biotechniques 34, 374-378 (2003).

57. Gu, Z., Gu, L., Eils, R., Schlesner, M. \& Brors, B. circlize Implements and enhances circular visualization in R. Bioinformatics 30, 2811-2812 (2014).

58. Thurmond, J. et al. FlyBase Consortium. FlyBase 2.0: the next generation Nucleic Acids Res. 47, D759-D765 (2019).

59. Yu, G., Wang, L. G., Han, Y. \& He, Q. Y. clusterProfiler: an R package for comparing biological themes among gene clusters. OMICS 16, 284-287 (2012).

60. Szklarczyk, D. et al. STRING v11: protein-protein association networks with increased coverage, supporting functional discovery in genome-wide experimental datasets. Nucleic Acids Res. 47, D607-D613 (2019).

61. Shannon, P. et al. Cytoscape: a software environment for integrated models of biomolecular interaction networks. Genome Res. 13, 2498-2504 (2003). 